\title{
Etiology of neonatal cholestasis after emerging molecular diagnostics
}

\author{
Huanhuan Wang ${ }^{1 \wedge}$, Lin Yang ${ }^{2}$, Jin Wang $^{1}$ \\ ${ }^{1}$ Division of Neonatology, Children's Hospital of Fudan University, National Children's Medical Center, Shanghai, China; ${ }^{2}$ Department of \\ Endocrinology and Inherited Metabolic Diseases, Children's Hospital of Fudan University, National Children's Medical Center, Shanghai, China \\ Contributions: (I) Conception and design: J Wang, H Wang; (II) Administrative support: J Wang; (III) Provision of study materials or patients: J \\ Wang, H Wang; (IV) Collection and assembly of data: J Wang, H Wang; (V) Data analysis and interpretation: All authors; (VI) Manuscript writing: \\ All authors; (VII) Final approval of manuscript: All authors. \\ Correspondence to: Jin Wang, MD. Division of Neonatology, Children's Hospital of Fudan University, National Children's Medical Center, 399 \\ Wanyuan Road, Minhang District, Shanghai 201102, China. Email: drwangjin@fudan.edu.cn.
}

Background: In the pediatric group, most cholestatic patients had disease onset at 0-3 months of age, and more and more are found to have specific genetic defects after failing to obtain a definite diagnosis by routine evaluation. To investigate the etiological diagnosis for the newborns with cholestasis during the neonatal period after emerging molecular tests comprehensively.

Methods: We conducted a retrospective study to evaluate clinical characteristics, etiologies and outcomes in infants with neonatal cholestasis after emerging molecular diagnostics from January 1st to December 31st, 2019 in Children's Hospital of Fudan University.

Results: There were 160 cases of neonatal cholestasis with mean gestational age (GA) $32.6 \pm 4.8$ weeks and birth weight $(\mathrm{BW}) 1,880 \pm 991 \mathrm{~g}$, composing $3.4 \%$ of total neonatal admissions in 2019 . Overall $97.5 \%(\mathrm{n}=156)$ patients had a definite diagnosis, including 9 obtaining a genetic diagnosis after adding molecular test in routine evaluation, which made the diagnosis rate for cholestasis increased by $5.6 \%$. The most common etiology of cholestasis in the neonatal period was parenteral nutrition-associated cholestasis (PNAC) 48.8\% $(\mathrm{n}=78)$, followed by cardiovascular and circulatory disorders $18.1 \%$, biliary anatomic obstruction $12.5 \%$, infection $8.7 \%$ and genetic disorders $5.6 \%$. PNAC and biliary anatomic obstruction were the most common etiology of cholestasis for preterm and term infants respectively. The mortality rate is $2.5 \%(\mathrm{n}=4)$ and $91.9 \%$ $(\mathrm{n}=147)$ patients totally recovery or improve in follow-up.

Conclusions: The causes of cholestasis in neonates are complicated, molecular diagnostics can improve the etiological diagnosis for newborns with cholestasis. But still, quite amount of causes are remediable and transient during the neonatal period, gene test may help to rule out genetic causes and enhance confidence in judging prognosis.

Keywords: Neonatal cholestasis; hyperbilirubinemia; etiology; molecular diagnosis

Submitted Oct 23, 2021. Accepted for publication Dec 30, 2021.

doi: $10.21037 / \mathrm{tp}-21-503$

View this article at: https://dx.doi.org/10.21037/tp-21-503

^ ORCID: 0000-0002-5006-629X. 


\section{Introduction}

Cholestatic jaundice affects approximately 1 in every 2,500 to 5,000 term infants and is thus infrequently observed by most providers when taking care of infants (1). Neonatal cholestasis is never physiological but rather a sign of hepatobiliary and/or metabolic disorders, some of which might be fatal if it is not identified and treated in time. Timely evaluation is essential for identifying those causes amenable to treatment and to offer accurate prognosis. The etiologic diversity of neonatal/infantile cholestasis has been described previously (2). The most commonly identifiable etiologies are biliary atresia (BA) $(25-35 \%)$, genetic intrahepatic cholestasis (25\%), and metabolic diseases (20\%), other risk factors included small for gestational age (SGA), neonatal surgery, alloimmune hemolytic disease and a history of some neonatal injurious event [asphyxia, sepsis, total parenteral nutrition (TPN), etc.] (3-7).

Recent studies in the understanding of the molecular basis of cholestatic syndromes can realize the classification of these syndromes, and it has offered an opportunity for the development of diagnostic methods that take into account the genetic makeup of neonatal/infantile intrahepatic cholestasis $(4,8)$. More and more cholestatic patients who failed to obtain a definite diagnosis by routine checking have been found to have specific genetic defects $(4,9)$, especially in the pediatric group (10).

As one of the largest medical centers for pediatric liver disease in China, our patients are distributed from all parts of China (11). Wang et al. found that most cholestatic patients had disease onset at $0-3$ month of age and about one-third obtained a genetic diagnosis. The rate of positive genetic diagnosis in patients with disease onset at $0-3$ month of age was higher than that onset at 4 months of age or later (12). But the situation of cholestasis in neonates is not clear.

To further investigate cholestatic patients with disease onset in the neonatal period, we conducted this study to evaluate clinical characteristics, etiology and outcomes in these infants after emerging molecular diagnostics in our neonatal intensive care unit (NICU).

We present the following article in accordance with the STROBE reporting checklist (available at https:// tp.amegroups.com/article/view/10.21037/tp-21-503/rc).

\section{Methods}

The study was conducted in accordance with the Declaration of Helsinki (as revised in 2013). The study was approved by Ethical Review Board of Children's Hospital of Fudan University [No.: (2021) 290] and individual consent for this retrospective analysis was waived. This study was to evaluate the incidence and potential causes of cholestasis for all the NICU admission newborns from January 1st to December 31st, 2019 at Children's Hospital of Fudan University. The flowchart of the patient inclusion and integration process is shown in Figure 1. The serum total and direct bilirubin from the initial blood biochemistry on admission was evaluated as inclusion criteria if it matches the definition, direct serum bilirubin level $>17.1 \mu \mathrm{mol} / \mathrm{L}(1 \mathrm{mg} / \mathrm{dL})$ when the total bilirubin is $<85.5 \mu \mathrm{mol} / \mathrm{L}(5 \mathrm{mg} / \mathrm{dL})$ or $>20 \%$ of the total bilirubin if the total bilirubin is $>85.5 \mu \mathrm{mol} / \mathrm{L}$ (4). And for all the cases without cholestasis on admission, the follow up of serum bilirubin would be done with the nutrition blood work every 1-3 weeks according to clinical requirements to find late-onset cases. The same definition was used as criteria. Once patients were noticed to have cholestasis whatever on admission or during the hospitalization, written informed consent would be obtained from the parents/guardian to get the blood sample for genetic analysis. Patients would be directly evaluated by panel sequencing, and Sanger sequencing was only used to confirm variants of interest (12).

The genetic tests were done using $2 \mathrm{~mL}$ of EDTAanticoagulated blood sample. DNA was extracted from peripheral blood. Clinical exome sequencing (CES) using the Agilent ClearSeq Inherited Disease panel kit (including 2,742 genes) was enriched from the fragments of patients' genomic DNA and performed on an Illumina HiSeq X10 (Illumina). The average on-target sequencing depth was $200 \times$ for CES (13). Variants of interest were validated by Sanger sequencing.

Except for the genetic tests, investigations for the etiology of neonatal cholestasis included infections, anatomic obstruction of the biliary system, endocrinopathies, metabolic disorders, cardiovascular and circulatory disorders, toxin and drug exposures, etc. (6). Nutritional management like Fat-soluble vitamins, medium-chain triglyceride (MCT) formula feeding is instead if breastmilk is not available. Satisfactory energy supplement, and symptomatic treatments such as Ursodesoxycholic acid and Compound Glycyrrhizin were commenced as well. The cause of neonatal cholestasis for each patient was confirmed by at least two of the three neonatologists.

SGA was defined as a birth weight $(B W)<10$ th percentile according to neonatal standard curves (14). Transient neonatal cholestasis is characterized by: early- 


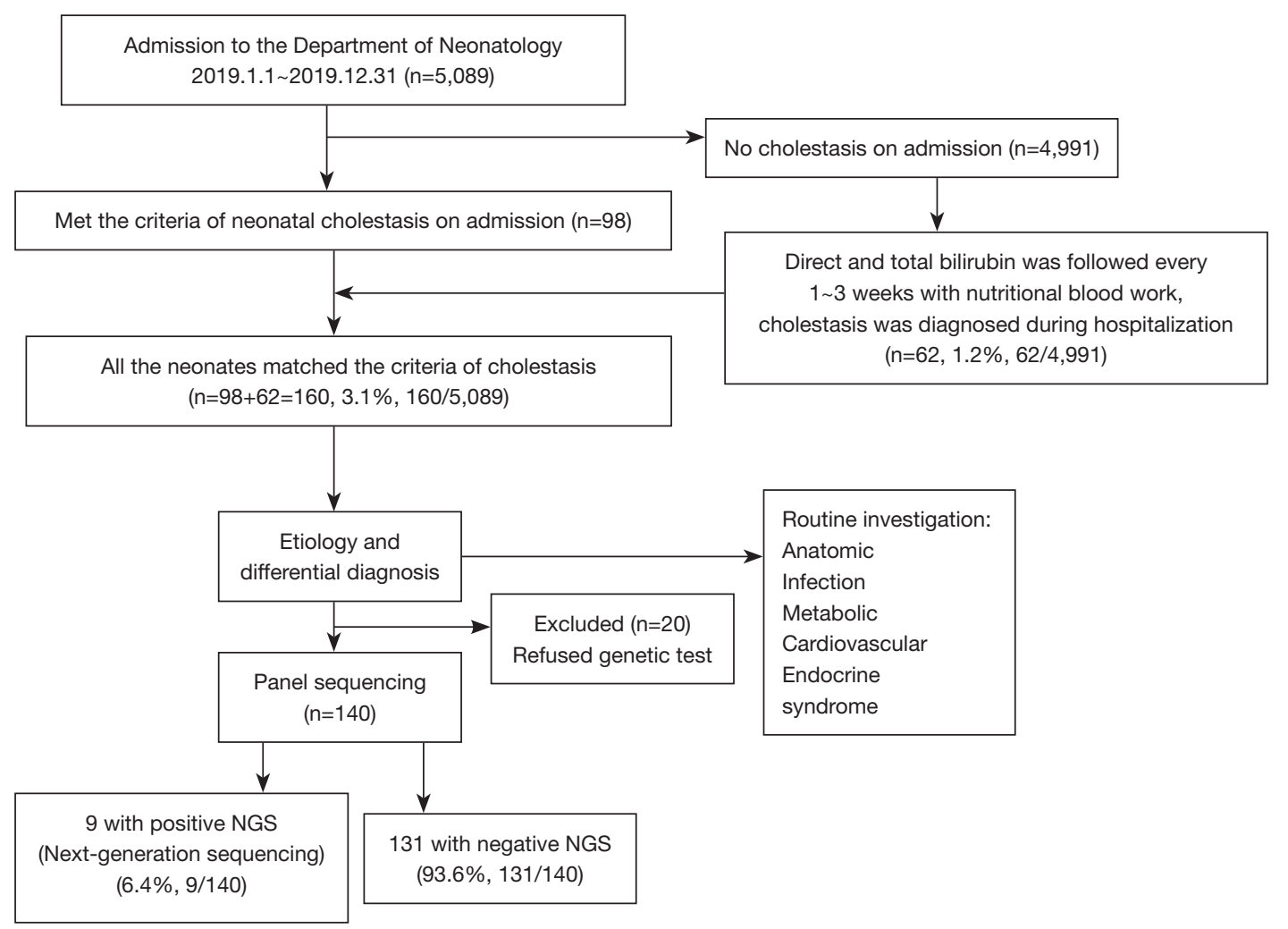

Figure 1 The flowchart of the patient inclusion and integration process. NGS, next-generation sequencing.

onset cholestasis, absence of a known cause of neonatal cholestasis, normalization of clinical and biochemical parameters during follow-up, and a history of some neonatal injurious event (asphyxia, sepsis, TPN, etc.) $(7,15)$. Parenteral nutrition-associated cholestasis (PNAC) was defined as neonates presented cholestasis after receiving $\mathrm{PN}$ for $\geq 14$ days and alternative causes of cholestasis were excluded, namely obstructive causes (e.g., extrahepatic BA), primary liver disease and metabolic liver disease. BA was confirmed by surgical exploration with an intraoperative cholangiogram. Red cell alloimmune hemolytic disease was identified by blood group, Coombs' and irregular antibody tests (5). Neonatal hemochromatosis was confirmed by the demonstration of extra-hepatic iron deposits sparing the reticuloendothelial system (16). The causes of neonatal cholestasis are based on the review from Feldman etc. (8).

\section{Statistical analysis}

Statistical analysis was performed using SPSS software (v. 22.0, SPSS Inc., Chicago, IL, USA). Continuous variables were summarized as mean $\pm \mathrm{SD}$, or median and interquartile range (IQR), or range. Categorical data were expressed as count and percentage (\%). Chi-square or Fisher's exact test for categorical variables and Mann-Whitney for continuous variables were used for comparison. For all analyses, significance was set at $\mathrm{P}<0.05$.

\section{Results}

\section{Clinical characteristics}

There were 160 cases of neonatal cholestasis, composing $3.14 \%(160 / 5,089)$ of total NICU admissions in 2019. There were 98 infants with cholestasis figured out on admission, and 62 cases $(1.24 \%)$ were noticed with cholestasis from the remaining 4,991 new admissions during hospitalization including 7 term and 55 preterm cases with GA from $25^{+2}$ to $36^{+3}$ weeks.

In all 160 cases, the male: female ratio is $1.8: 1$. The mean GA is $32.6 \pm 4.8$ weeks, in which $71.2 \%(\mathrm{n}=114)$ are GA $<37$ weeks. The mean BW is $1,880 \pm 991 \mathrm{~g}$ with $48.1 \%(\mathrm{n}=77)$ born with a $\mathrm{BW}<1,500 \mathrm{~g}$. The mean cholestasis onset age is $27 \pm 19$ days. Patients with SGA account for $18.8 \%(n=30)$. 


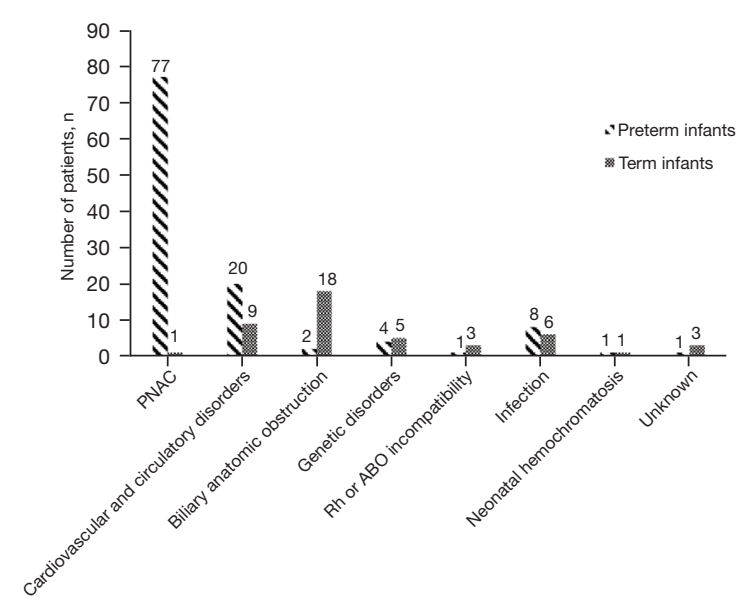

Figure 2 Etiology of neonatal cholestasis in term and preterm infants.

\section{Causes of neonatal cholestasis}

The most common etiology of cholestasis was PNAC $48.8 \%(\mathrm{n}=78)$, followed by cardiovascular and circulatory disorders $18.1 \%(n=29)$, biliary anatomic obstruction $12.5 \%(\mathrm{n}=20)$ (including surgery confirmed $\mathrm{BA}, \mathrm{n}=17)$, infection $8.7 \%$ [ $\mathrm{n}=14$, including cytomegalovirus (CMV), $\mathrm{n}=7$; enterovirus, $\mathrm{n}=4$; fungus, $\mathrm{n}=1$; mycoplasma, $\mathrm{n}=1$ and syphilis, $\mathrm{n}=1]$, genetic and metabolic disorders $5.6 \%(\mathrm{n}=9)$, post $\mathrm{Rh}$ or $\mathrm{ABO}$ incompatibility $2.5 \%(\mathrm{n}=4$, including $\mathrm{Rh}$ incompatibility, $\mathrm{n}=3$ and $\mathrm{ABO}, \mathrm{n}=1$ ), neonatal hemochromatosis $1.2 \%(\mathrm{n}=2)$, and unknown $2.5 \%(\mathrm{n}=4)$.

PNAC and biliary anatomic obstruction were the most common etiology of cholestasis for preterm and term infants respectively (Figure 2). There were $98.7 \%$ and $79.5 \%$ neonates with $\mathrm{GA}<37$ weeks and $\mathrm{BW}<1,500 \mathrm{~g}$ in neonates with PNAC respectively. Of all the 78 cases of PNAC, 33 cases belong to the 62 cases without this issue on admission and the initial diagnosis of PNAC was made during the follow-up nutrition blood work. Another 45 cases of PNAC were transferred from local hospitals had a much more complicated course of diagnosis and management.

The group of cardiovascular and circulatory disorders included both antenatal and postpartum events, and vascular malformation. There were $15 / 29$ cases in this category identified as SGA, all of them with a history of intrauterine growth retardation (IUGR) due to pregnancyinduced hypertension( $\mathrm{n}=8)$, Twin-to-twin transfusion syndrome $(n=4)$, selective IUGR $(n=2)$, and severe rotation of umbilical cord $(n=1)$, and almost all the cases had been noticed with reverse umbilical blood flow from intrauterine ultrasound. The cholestasis onset age in this group was 8 [6-10] days after birth. There were 4/29 defined as special circulatory disorders associating with portal vein or hepatic vessels malformation including hepatic hemangioma $(n=2)$, hepatic arteriovenous fistula $(n=1)$, and congenital portosystemic shunts $(\mathrm{n}=1)$.

There were a total of $87.5 \%(n=140)$ cases with genetic tests, in which 36 variants were picked up, but only 9 were validated by Sanger sequencing and considered as a positive genetic test for cholestasis, including Alagille syndrome $(\mathcal{F} A G 1, \mathrm{n}=2)$, pigment incontinence (IKBKG, $\mathrm{n}=2)$, Niemann-Pick disease, type C1 (NPC1, n=1), Coproporphyria (CPOX, $\mathrm{n}=1$ ) and Hyper-IgD Syndrome $(M V K, \mathrm{n}=1)$, Williams-Beuren syndrome $(\mathrm{n}=1)$, Down Syndrome (n=1) (Table S1).

There were 20 cases of decline genetic tests including 8 cases in the PNAC group, 2 in cardiovascular and circulatory disorders, 4 in Biliary anatomic obstruction, 2 in infection, 2 in transient cholestasis secondary to hemolysis, and 2 in the unknown group, while the other 2 in the unknown group had negative genetic tests.

\section{Prognosis}

The overall mortality was $2.5 \%(\mathrm{n}=4)$, and the rate of full recovery from cholestasis was $15.6 \%(n=25)$ at discharge. In the remaining 131 patients, $6.9 \%(\mathrm{n}=9)$ lost follow-up, $55.0 \%(\mathrm{n}=72)$ totally recovered from cholestasis and $38.2 \%$ $(\mathrm{n}=50)$ improved from cholestasis during one year's follow up. The 9 infants who lost follow-up included 4 PNAC, $3 \mathrm{BA}, 1$ infection and 1 hepatic arteriovenous fistula.

Compared with PNAC infants, non-PNAC ones had a lower proportion of preterm infants and infants with $\mathrm{BW}$ $<1,500 \mathrm{~g}(37 / 82,45.1 \%$ vs. $77 / 78,98.7 \%, \mathrm{P}<0.001)$ and (15/82, $18.3 \%$ vs. $62 / 78,79.5 \%, \mathrm{P}<0.001)$, early cholestasis onset age (12 vs. 39 days, $\mathrm{P}<0.001)$, early-onset age of elevated ALT (21 vs. 55 days, $\mathrm{P}<0.001$ ), and quicker improvement from cholestasis ( 38 vs. 76 days, $\mathrm{P}<0.001$ ) and shorter length of hospital stay (20 vs. 60 days, $\mathrm{P}<0.001)$ (Table 1).

Two dead infants in the PNAC group had negative results of genetic tests, necrotizing enterocolitis and sepsis were the radical causes for death respectively. Four cases in the PNAC group lost to follow up with an unknown outcome.

For the 29 infants in the group of cardiovascular and circulatory disorders, $93.1 \%(n=27)$ improved or recovered from cholestasis except 1 died at 13 days of life because of 
Table 1 PNAC and non-PNAC infants: clinical characteristics and outcomes

\begin{tabular}{lccc}
\hline & PNAC (N=78) & Non-PNAC (N=82) & P value \\
\hline GA, w & $29.1 \pm 2.6$ & $35.9 \pm 4.0$ & $<0.001$ \\
SGA & $10(12.8)$ & $20(24.4)$ & 0.061 \\
GA $<37$ w & $77(98.7)$ & $37(45.1)$ & $<0.001$ \\
BW, g & $1,255 \pm 512$ & $15(18.3)$ & $<0.001$ \\
BW $<1,500 \mathrm{~g}$ & $62(79.5)$ & $17[6-28]$ & $<0.001$ \\
Admission age, d & $28[0-53]$ & $12[5-22]$ & $<.065$ \\
cholestasis onset age, d & $39[28-54]$ & $21[13-33]$ & $<0.001$ \\
onset age of elevated ALT, d & $55[44-72]$ & $55(67.1)$ & $<0.001$ \\
Elevated ALT, $n$ (\%) & $45(57.7)$ & $38[29-60]$ & $<.260$ \\
Age of cholestasis improved, d & $76[62-97]$ & $20[12-37]$ & $<0.001$ \\
Duration of hospital stay, d & $60[30-81]$ & $<0.001$ & $<$
\end{tabular}

Values are given in $\mathrm{n}(\%)$, mean $\pm \mathrm{SD}$ or median and interquartile range [IQR]. Comparisons of categorical variables were performed with Chi square test or Fisher's exact test. Mann-Whitney test was applied for comparisons of continuous variables. PNAC, parenteral nutritionassociated cholestasis; GA, gestational age; SGA, small for GA; BW, birth weight; ALT, Alanine aminotransferase.

hemorrhagic shock, and 1 with hepatic arteriovenous fistula lost follow-up.

For the 20 infants with biliary anatomic obstruction, 85.0\% ( $\mathrm{n}=17)$ with BA got better after Kasai surgical operation until one year follow-up. The rest 3 were suspected as BA by positive findings of ultrasound and/or hepatobiliary radionuclide scans, but parents refused further cholangiogram and lost follow-up.

In the infection group, only 1 with enterovirus infection lost to follow-up with quite severe liver dysfunction and the rest $92.8 \%(n=13)$ recovered completely or got better during follow-up.

There were 4 cases secondary to alloimmune hemolytic disease, $3 \mathrm{Rh}$ and $1 \mathrm{ABO}$ incompatibility, all getting better. 2 infants with neonatal hemochromatosis, one died of liver failure, the other with Down syndrome improved from cholestasis. All the 9 infants with positive genetic tests got better or stable during follow-up.

\section{Discussion}

Cholestasis is not a common situation in newborn period, while more and more studies demonstrated it may be an important evaluation part for the newborns in NICU. The overall incidence of the newborn with cholestasis in NICU from our data is $3.14 \%(160 / 5,089)$, which is greatly higher than the incidence in live births (17). Except for the part admitted for cholestasis, the incidence of cholestasis confirmed during the stay of hospitalization is $1.24 \%$ $(62 / 4,991)$. This is still ten times higher than the overall incidence in a live birth, and the evaluation for cholestasis is necessary for the population in NICU, and the follow-up screening could be included in the nutrition blood work.

PNAC was the most common causative factor in newborn cholestasis cases from our data, mainly involves very and extremely low BW, and post-surgery premature infants. These cases had lifesaving TPN, and some of them also suffered from confirmed risk factors for cholestasis, such as lack of enteral feeds, intestinal surgery, experiences of sepsis and antibiotics. PNAC is an early stage of the spectrum of $\mathrm{PN}$-associated liver disease which ranges from mild and reversible cholestasis to end-stage liver disease (18). The definition of PNAC is varied, but the usual criteria are direct bilirubin of $2 \mathrm{mg} / \mathrm{dL}$ or greater while being on PN for 14 or more days (19). Therefore, the diagnosis was considered only after TPN therapy for more than two weeks and the bilirubin parameter wasn't matched cholestasis before TPN was initiated. In our data, except one term infant with congenital diaphragm hernia presented cholestasis was considered as PNAC, most of totally 78 cases of PNAC were preterm infants with the average GA of $29.1 \pm 2.6$ weeks, who were recognized as the high-risk population of PNAC. The comparison between PNAC and non-PNAC showed the cholestasis onset age 
was much older, TPN duration was much longer, and the average GA was smaller in the PNAC group. In this group, nutrition blood work follow-up every one to three weeks was routine and exercisable, and the results for PNAC were almost detected as early as possible. The overall prognosis of PNAC was better if it was detected in the early stage. The incidence of PNAC has been improved compared with our previous data, which may be benefited from the overall nutritional management and the adoption of SMOF (lipid emulsion consisting of soybean oil, medium-chain triglycerides, olive oil, and fish oil) (20).

The second cause of cholestasis in our data was the category of cardiovascular and circulatory disorders. It included both antenatal and postpartum events, there were 15 in 29 cases in this category identified as SGA according to the Fenton growth chart (14). Except for IUGR, they all required TPN after birth due to low BW and feeding intolerance and abdominal distention, while cholestasis onset age in this group was much earlier than it in the PNAC group, 8 [6-10] vs. 39 [28-54] days. Some units considered this as transient neonatal cholestasis with additional risk factors beyond PN. Postpartum events more likely affected term or near-term infants. Cholestasis was the sign of liver damage, as one of multiple organs damage (7). There were 4/29 in total defined as special circulatory disorders associating with portal vein or hepatic vessels in our data. These are not commonly defined causes for cholestasis, but in the neonatal population, we do recognize these should be integrated into the regular investigation of neonatal cholestasis to rule out congenital vessel malformations. These could be treatable clinical disorders if they can be found in the early-stage, Doppler ultrasound as a non-invasive screening is a first-line option. The further intervention requirements need clinical and imaging assessments, some could be asymptomatic and no needs for treatment except follow-up $(21,22)$.

Biliary anatomic obstruction was the third cause for neonatal cholestasis as expected. We had a total of 20 cases in this category, $90.0 \%(\mathrm{n}=18)$ were term infants. There were $85.0 \%(\mathrm{n}=17)$ confirmed as BA by cholangiogram in surgery and had Kasai operations as a reasonable option. All the cases were admitted to NICU because of elevated conjugated bilirubin and/or pale stools and the average diagnostic time was 35 days (13-51 days) of life, within one week after admission. The stool color card as a screening test in China has helped to move up the diagnostic time of BA to the neonatal period (23). Early operation within two months of age helped to improve the overall prognosis (24).
Infections such as CMV (7/14), enterovirus (4/14), fungus (1/14), mycoplasma (1/14) and syphilis (1/14) were all reported as an etiology of neonatal cholestasis (25). Maternal history, serum antibody, DNA of pathogen and metagenomic next-generation sequencing (mNGS) were tested and collected for diagnosis. The infection of enterovirus was most concerned because of the multiple organ dysfunction and the possibility of poor outcome. The other types of infection, most belonging to TORCH were well understood, the outcomes are various according to different pathogens and different infection time points (26-29). Fortunately, all the outcomes in our cases with infection were benign except one of enterovirus lost to follow up.

Transient neonatal cholestasis secondary to $\mathrm{Rh}$ or ABO hemolysis was another optimum cause in neonatal cholestasis. Most cases would present self-limited and selfrecover and no special therapy was always required. It has been studied that in neonates with severe erythroblastosis fetalis and Rh or ABO incompatibility (5), and G6PD as well (30).

Neonatal hemochromatosis is a gestational alloimmune liver disease in neonatal period, which is a very rare disease and it could be misdiagnosed without pathology or demonstration of siderosis by magnetic resonance imaging (MRI) imaging $(31,32)$. Extrahepatic siderosis in the pancreas, myocardium, thyroid and minor salivary gland is a characteristic feature of neonatal hemochromatosis. Although cascade of events leads to acute liver failure and neonatal death, it was treated with intravenous immune globulin (IVIG) successfully (33).

Genetic and metabolic disorders were considered as one of the most common causes in $0-3$ months infants. This was the original intention to design this project in the neonatal period. We collected 140 cases who have genetic tests, in which 36 variants were picked up, but only 9 were validated by Sanger sequencing and considered as a positive genetic test for cholestasis. The previous report obtained a molecular diagnosis with cholestasis onset at $0-3$ months of age was $37.2 \%$ (12). In our data, the overall incidence of neonatal cholestasis with a genetic diagnosis was only $6.43 \%(9 / 140)$ and the $7 A G 1$ was the one listed in the most common genes. The reason for the difference between neonates and $0-3$ months is considered as below: first, most mild PNAC diagnosed in NICU were followed up by neonatologists, and not referred to hepatologists. Second, the numbers could be underestimated as the panel sequencing was recommended to all cases once cholestasis 
onset, but there were still 20 in 160 cases refused by the parents for different reasons, and some of them recovered completely in follow-up, while some were discharged without medical advice and follow-up. Although the genetic diagnosis wasn't picked up as many cases as we expected, it did help rule out the reason for genetics in the other categories.

After genetic test commenced for neonates with cholestasis, the total diagnosed rate for cholestasis was $97.5 \%(156 / 160)$ from our data, which is much better than ten years ago when we had no genetic test. Our study in 2012 presented 7 -year data, 3/35 unknown and 3/35 with unclear genetic or metabolic problems in term infants, and 13/141 unknown and 2/141 with unclear genetic or metabolic problems in preterm infants with cholestasis, the total diagnosed rate was only $85.8 \%$ (151/176) (34). And we did recognize that the other clear etiologies of neonatal cholestasis were quite similar if deducting genetic and metabolic causes, now $91.9 \%(147 / 160)$ vs. $85.8 \%$ (151/176). The importance of genetic test does not only provide a diagnostic pattern, but it may also help for the therapy in the future (8).

\section{Conclusions}

The causes of cholestasis in neonates are complicated. The molecular diagnostic test can improve the diagnosis for these infants. Although inherited intrahepatic cholestasis is one of the most common causes in young infants, we should realize that there are many remediable causes for cholestasis in the neonatal period as well. PNAC and BA were the most common cause of cholestasis for preterm and term infants respectively, and transient cholestasis secondary to hemolysis, cardiovascular and circulatory disorder, congenital structure anomalies of the hepatobiliary system and gestational alloimmune liver diseases are also special causes in the neonatal period. Gene tests may help to rule out genetic causes and enhance confidence in judging prognosis.

\section{Acknowledgments}

We would like to thank all the family who participated in this study. We greatly appreciate all the staff from the neonatal department of Children's Hospital of Fudan University taking care of the patients and providing assistance during the research process. The authors also thank Dr. Meijian Zhao from Southlake Regional Health
Center, CA and assistant professor Zhiguo Zhou from University of Central Missouri, USA for embellishing and editing.

Funding: None.

\section{Footnote}

Reporting Checklist: The authors have completed the STROBE reporting checklist. Available at https:// tp.amegroups.com/article/view/10.21037/tp-21-503/rc

Data Sharing Statement: Available at https://tp.amegroups. com/article/view/10.21037/tp-21-503/dss

Peer Review File: Available at https://tp.amegroups.com/ article/view/10.21037/tp-21-503/prf

Conflicts of Interest: All authors have completed the ICMJE uniform disclosure form (available at https://tp.amegroups. com/article/view/10.21037/tp-21-503/coif). The authors have no conflicts of interest to declare.

Ethical Statement: The authors are accountable for all aspects of the work in ensuring that questions related to the accuracy or integrity of any part of the work are appropriately investigated and resolved. The study was conducted in accordance with the Declaration of Helsinki (as revised in 2013). The study was approved by Ethical Review Board of Children's Hospital of Fudan University [No.: (2021) 290] and individual consent for this retrospective analysis was waived.

Open Access Statement: This is an Open Access article distributed in accordance with the Creative Commons Attribution-NonCommercial-NoDerivs 4.0 International License (CC BY-NC-ND 4.0), which permits the noncommercial replication and distribution of the article with the strict proviso that no changes or edits are made and the original work is properly cited (including links to both the formal publication through the relevant DOI and the license). See: https://creativecommons.org/licenses/by-nc-nd/4.0/.

\section{References}

1. Fawaz R, Baumann U, Ekong U, et al. Guideline for the Evaluation of Cholestatic Jaundice in Infants: Joint Recommendations of the North American Society for Pediatric Gastroenterology, Hepatology, and Nutrition 
and the European Society for Pediatric Gastroenterology, Hepatology, and Nutrition. J Pediatr Gastroenterol Nutr 2017;64:154-68.

2. Balistreri WF, Bezerra JA, Jansen P, et al. Intrahepatic cholestasis: summary of an American Association for the Study of Liver Diseases single-topic conference. Hepatology 2005;42:222-35.

3. Balistreri WF, Bezerra JA. Whatever happened to "neonatal hepatitis"? Clin Liver Dis 2006;10:27-53, v.

4. Feldman AG, Sokol RJ. Neonatal Cholestasis. Neoreviews 2013;14:10.1542/neo.14-2-e63. doi:10.1542/ neo.14-2-e63

5. Smits-Wintjens VE, Rath ME, Lindenburg IT, et al. Cholestasis in neonates with red cell alloimmune hemolytic disease: incidence, risk factors and outcome. Neonatology 2012;101:306-10.

6. Lane E, Murray KF. Neonatal Cholestasis. Pediatr Clin North Am 2017;64:621-39.

7. Champion V, Carbajal R, Lozar J, et al. Risk factors for developing transient neonatal cholestasis. J Pediatr Gastroenterol Nutr 2012;55:592-8.

8. Feldman AG, Sokol RJ. Neonatal cholestasis: emerging molecular diagnostics and potential novel therapeutics. Nat Rev Gastroenterol Hepatol 2019;16:346-60.

9. Stormon MO, Dorney SF, Kamath KR, et al. The changing pattern of diagnosis of infantile cholestasis. J Paediatr Child Health 2001;37:47-50.

10. Mieli-Vergani G, Howard ER, Mowat AP. Liver disease in infancy: a 20 year perspective. Gut 1991;Suppl:S123-8.

11. Wang NL, Lu YL, Zhang P, et al. A Specially Designed Multi-Gene Panel Facilitates Genetic Diagnosis in Children with Intrahepatic Cholestasis: Simultaneous Test of Known Large Insertions/Deletions. PLoS One 2016;11:e0164058.

12. Wang NL, Lu Y, Gong JY, et al. Molecular findings in children with inherited intrahepatic cholestasis. Pediatr Res 2020;87:112-7.

13. Yang L, Kong Y, Dong X, et al. Clinical and genetic spectrum of a large cohort of children with epilepsy in China. Genet Med 2019;21:564-71.

14. Fenton TR, Kim JH. A systematic review and metaanalysis to revise the Fenton growth chart for preterm infants. BMC Pediatr 2013;13:59.

15. Ciocca M, Alvarez F. Transient neonatal cholestasis. Arch Argent Pediatr 2011;109:163-6.

16. Shanmugam NP, Bansal S, Greenough A, et al. Neonatal liver failure: aetiologies and management--state of the art.
Eur J Pediatr 2011;170:573-81.

17. Zollner G, Trauner M. Mechanisms of cholestasis. Clin Liver Dis 2008;12:1-26, vii.

18. Satrom K, Gourley G. Cholestasis in Preterm Infants. Clin Perinatol 2016;43:355-73.

19. Carter BA, Shulman RJ. Mechanisms of disease: update on the molecular etiology and fundamentals of parenteral nutrition associated cholestasis. Nat Clin Pract Gastroenterol Hepatol 2007;4:277-87.

20. Qian T, Zhang R, Zhu L, et al. Very low birth weight preterm infant complications where parenteral nutrition is soy or fish oil-based: A retrospective study in Shanghai. Asia Pac J Clin Nutr 2020;29:552-7.

21. Uike K, Nagata H, Hirata Y, et al. Effective shunt closure for pulmonary hypertension and liver dysfunction in congenital portosystemic venous shunt. Pediatr Pulmonol 2018;53:505-11.

22. Iacobas I, Phung TL, Adams DM, et al. Guidance Document for Hepatic Hemangioma (Infantile and Congenital) Evaluation and Monitoring. J Pediatr 2018;203:294-300.e2.

23. Kong YY, Zhao JQ, Wang J, et al. Modified stool color card with digital images was efficient and feasible for early detection of biliary atresia-a pilot study in Beijing, China. World J Pediatr 2016;12:415-20.

24. Zheng J, Ye Y, Wang B, et al. Biliary atresia screening in Shenzhen: implementation and achievements. Arch Dis Child 2020;105:720-3.

25. Ananth R. Neonatal Cholestasis: A Primer of Selected Etiologies. Pediatr Ann 2018;47:e433-9.

26. Leung KKY, Hon KL, Yeung A, et al. Congenital infections in Hong Kong: an overview of TORCH. Hong Kong Med J 2020;26:127-38.

27. Neu N, Duchon J, Zachariah P. TORCH infections. Clin Perinatol 2015;42:77-103, viii.

28. Muehlenbachs A, Bhatnagar J, Zaki SR. Tissue tropism, pathology and pathogenesis of enterovirus infection. J Pathol 2015;235:217-28.

29. Pappas PG, Kauffman CA, Andes DR, et al. Clinical Practice Guideline for the Management of Candidiasis: 2016 Update by the Infectious Diseases Society of America. Clin Infect Dis 2016;62:e1-50.

30. Ben Fredj D, Barro C, Joly P, et al. Transient liver injury and severe neonatal cholestasis in infant with glucose6-phosphate dehydrogenase deficiency due to a new mutation. Arch Pediatr 2019;26:370-3.

31. Chavhan GB, Kamath BM, Siddiqui I, et al. Magnetic resonance imaging of neonatal hemochromatosis. Pediatr 
Radiol 2022;52:334-9.

32. Alenezi K, Kamath BM, Siddiqui I, et al. Magnetic Resonance Imaging Findings in Neonatal Hemochromatosis. J Pediatr Gastroenterol Nutr 2018;66:581-7.

33. Jimenez-Rivera C, Gupta A, Feberova J, et al. Successful treatment of neonatal hemochromatosis as gestational alloimmune liver disease with intravenous immunoglobulin. J Neonatal Perinatal Med 2014;7:301-4.

34. Wang J, Zhang R, An N, et al. Clinical features and etiology of cholestasis in neonates. Zhonghua Yi Xue Za Zhi 2012;92:1259-63.
Cite this article as: Wang H, Yang L, Wang J. Etiology of neonatal cholestasis after emerging molecular diagnostics. Transl Pediatr 2022;11(3):359-367. doi: 10.21037/tp-21-503 